\title{
Evaluation of PTEN loss and PIK3CA mutations and their correlation with efficacy of trastuzumab treatment in HER2-positive metastatic breast cancer: A retrospective study (KBC-SG 1001)
}

\author{
REIKI NISHIMURA $^{1}$, NOBUYUKI ARIMA ${ }^{2}$, SATOSHI TOYOSHIMA $^{3}$, YASUYO OHI $^{4}$, KEISEI ANAN $^{5}$, \\ YASUAKI SAGARA ${ }^{6}$, SHOSHU MITSUYAMA ${ }^{5}$ and KAZUO TAMURA ${ }^{7}$
}

\begin{abstract}
Departments of ${ }^{1}$ Breast and Endocrine Surgery and ${ }^{2}$ Pathology, Kumamoto City Hospital, Kumamoto;
${ }^{3}$ Department of Pathology, Kitakyushu Municipal Medical Center, Kitakyushu; ${ }^{4}$ Department of Pathology,

Hakuaikai Sagara Hospital, Kagoshima; ${ }^{5}$ Department of Surgery, Kitakyushu Municipal Medical Center, Kitakyushu;

${ }^{6}$ Department of Breast/Thyroid Gland, Hakuaikai Sagara Hospital, Kagoshima; ${ }^{7}$ Division of Medical Oncology,

Hematology and Infectious Diseases, Department of Medicine, Fukuoka University Hospital, Fukuoka, Japan
\end{abstract}

Received May 29, 2012; Accepted July 27, 2012

DOI: $10.3892 /$ mco.2012.10

\begin{abstract}
Trastuzumab (T) has contributed to improving the prognosis of human epidermal growth factor receptor 2 (HER2)-positive breast cancer. Although some patients have been unresponsive or resistant to T. Loss of phosphatase and tensin homolog (PTEN) deleted on chromosome 10, PIK3CA mutation and p95HER 2 expression have been reported to potentially be responsible for the poor response to T. This is a small-scale pilot study to be followed by a large-scale investigation examining the association between the biomarkers and clinical response. Based on the response to T, patients were divided into 3 groups in terms of progression-free survival (PFS): PFS $>8$ months (group $A, n=15$ ), 3-8 months (group $\mathrm{B}, \mathrm{n}=7$ ) and PFS $<3$ months (group $\mathrm{C}, \mathrm{n}=11$ ). PTEN protein expression was detected by immunohistochemistry and PIK3CA mutation by direct sequencing. The median age was 61,60 and 47 years in groups A, B and C, respectively, with statistically significant differences among the groups. No additional patient background factors differed between the groups. A decreased PTEN expression (H score, $<100)$ was observed in 33.3 and $72.7 \%$ of patients in groups A and C, respectively. PTEN loss was slightly correlated with poor response to T. PIK3CA mutation frequency in exons $9 / 20$ was $33.3 \%$ in group A and $27.3 \%$ in group C, with no significant correlation between PIK3CA mutation and clinical response. In this small-scale pilot study, a weak correlation
\end{abstract}

Correspondence to: Dr Reiki Nishimura, Department of Breast and Endocrine Surgery, Kumamoto City Hospital, 1-1-60 Kotoh, Higashi-ku, Kumamoto 862-8505, Japan

E-mail: nishimura.reiki@cityhosp-kumamoto.jp

Key words: breast cancer, PTEN, PIK3CA, HER2-positive was demonstrated between PTEN loss and poor response to $\mathrm{T}$. This potential correlation is likely to be confirmed in the planned large-scale study, while the association of PIK3CA mutation and p95HER 2 expression with poor response to $\mathrm{T}$ also requires examination.

\section{Introduction}

The overexpression of the human epidermal growth factor receptor 2 (HER2) gene is observed in 20-30\% of human breast cancer patients (1). Treatment for this type of breast cancer has markedly improved since the approval of the monoclonal antibody trastuzumab (T), and the combination of lapatinib with capecitabine, also available since June 2009 in Japan.

However, p95HER2 protein expression, loss of phosphatase and tensin homolog (PTEN) deleted on chromosome 10 and PIK3CA gene mutation have demonstrated a resistance to T treatment $(2,3)$. p95HER2 is the carboxyl terminal fragment (CTF) of HER2, while p95HER2 lacked the T binding site. Therefore, p95HER2 expression in HER2-positive breast cancer appeared to be involved in T resistance. p95HER2 protein was co-expressed with wild-type HER2 in 14-60\% of the HER2-positive breast cancer cases (4,5). In addition, PTEN is a cancer suppressor gene, known to undergo mutations in various types of cancer. It is located on chromosome 10q23.3, coding for the protein created from the 403 amino acid residue that acts as a phospholipid phosphatase. PTEN inhibits signal transmission of the phosphatidyl inositol (PI-3) kinase downstream. Due to mutation of the PTEN gene, decreased PTEN function activates the P13K downstream signal transmission system that subsequently affects cell functions, such as proliferation, apoptosis and cell infiltration. This PTEN protein loss was observed in 20.5-76.3\% of the HER2-positive breast cancer cases (6-10), demonstrating an existing correlation between the treatment effect of $\mathrm{T}$ and the level of PTEN protein expression $(6,7)$. 
PIK3CA mutation is observed in 10.3-37.5\% of the HER2positive breast cancer cases (9-12). Exons 9 (E542K, E545K) and $20(\mathrm{H} 1047 \mathrm{R})$ are the hot spots for mutation $(13,14)$, demonstrating that the effect of $\mathrm{T}$ treatment was affected by these mutations (10). However, the effect of lapatinib treatment has been proven not to be correlated with the p95HER2 expression levels or PTEN proteins, or the status of PIK3CA gene mutation $(8,11,12,15)$. These biological features are worth examining in terms of their treatment response to $\mathrm{T}$ or lapatinib.

The Kyushu Breast Cancer Study Group (KBC-SG) is conducting a prospective clinical study to investigate the correlation between the antitumor effect of lapatinib + capecitabine concomitant treatment and the following biomarkers: p95HER2 expression, PTEN loss and PIK3CA mutation in HER2-positive breast cancer patients pre-treated with T. Prior to conducting a prospective clinical study, the evaluation methods of these biomarkers needed to be established by using stored tumor tissue samples of HER2-positive breast cancer patients. The frequency of p95HER2 expression, PTEN loss and PIK3CA mutation in relation to $\mathrm{T}$ treatment are also of notable interest. The present study is a feasibility study aiming to validate the applicability of our technique in a prospective clinical study.

Despite the original plan, considering the results of a VeraTag $^{\text {TM }}$ assay (16) the use of immunohistochemistry for the p95HER2 protein test was deemed to be unnecessary in this preliminary study.

\section{Patients and methods}

Patient characteristics. HER2-positive tumor samples were obtained from the Kumamoto City Hospital (Kumamoto, China), the Hakuaikai Sagara Hospital (Kagoshima, China) and the Kitakyushu Municipal Medical Center (Kitakyushu, China). The present study was approved by the corresponding Institutional Review Boards. The subjects were patients with HER2-positive breast cancer, with documented progression on at least one T-containing regimen in the metastatic setting, meeting the following criteria: i) females diagnosed with invasive breast cancer by histological examination; ii) patients with overexpression of HER2, i.e. the staining score detected by immunohistochemistry (IHC) being $3+$, or HER2 gene amplification being confirmed by fluorescence in situ hybridization (FISH); iii) patients with a T treatment history and obtainable detailed clinical data; iv) availability of formalin-fixed and paraffin-embedded tissues of primary tumors and v) patients willing to participate in this study.

Methods. To compare the biomarker results with response to $\mathrm{T}$ treatment, PTEN loss and PIK3CA mutation were studied in 3 groups, on the basis of treatment responses $(17,18)$. The patients were divided as follows: i) group A, responders to $\mathrm{T}$ treatment [progression-free survival time (PFS) of at least 8 months] $\geq 10$ patients; ii) group $\mathrm{B}$, worsening in the early phase of $\mathrm{T}$ treatment (PFS from 3 to 8 months of $\mathrm{T}$ treatment), with the highest possible number of group B subjects being collected, 0-10 patients; iii) group $\mathrm{C}$, non-responders to $\mathrm{T}$ treatment (PFS $<3$ months of $\mathrm{T}$ treatment or relapse during adjuvant $\mathrm{T}$ treatment), $\geq 10$ patients.
The following indices were investigated in this study: i) methods to evaluate PTEN expression, ii) frequency of PTEN loss and PIK3CA mutation with regard to response to T treatment.

Evaluation methods of PTEN. The immunohistochemical staining for PTEN protein in tumor cells was evaluated using anti-human PTEN mouse monoclonal antibodies (clone 6H2.1, M3627; Dako, Copenhagen, Denmark), while the EnVision ${ }^{\mathrm{TM}}$ FLEX+ kit mouse linker (Dako) was used to detect the protein, due to the weak PTEN protein expression level in the standard expression system. Normal breast epithelium or vascular endothelium known to express normal PTEN was used as the positive control. The staining conditions, such as the dilution factor, as well as the pre-treatment conditions were examined according to the manufacturer's instructions. The three pathologists evaluated the PTEN expression independently. The $\mathrm{H}$ score was used to evaluate and score the expression levels (19). The staining intensity (0-3) was evaluated using the semantic anchors: negative $(0)$, weak $(1+)$, moderate $(2+)$ and strong (3+). To obtain percentages the area occupying the positive cells over the cells was estimated.

Investigation of PIK3CA mutation. DNA was extracted from formalin-fixed and paraffin-embedded blocks. Analysis of the mutations of the PIK3CA gene was performed using the direct sequence method. As previously mentioned, the base sequences in exons 9 and 20, which are the hot-spots of the PIK3CA gene mutation (13), were decoded and evaluated.

Statistical analysis. Statistical comparisons between groups were conducted using the Chi-square and Fisher's exact tests. The Wilcoxon's (non-parametric) test was used to compare the mean values for age, and the signal ratio of HER2-FISH, disease-free survival (DFI) and PFS. A two-sided $\mathrm{P}<0.05$ was considered to indicate a statistically significant difference.

\section{Results}

Background characteristics of patients. The number of patients was as follows: group A, 15; group B, 7 and group C, 11. The background characteristics of these patients are presented in Table I. The median age at registration was 60 years for groups $\mathrm{A}$ and $\mathrm{B}$, and 47 years for group $\mathrm{C}$, suggesting a significant age difference. No difference was detected, however, in the 3 groups regarding tumor diameter, lymph-node metastasis, stage, DFI, type of surgery, HER2 status by IHC, signal count by FISH and the T treatment period. Significant differences were detected between groups $\mathrm{A}$ and $\mathrm{C}$ and groups $\mathrm{B}$ and $\mathrm{C}$ regarding the PFS of the T treatment. In the ER, 81.8\% of the patients in group $\mathrm{C}$ were negative, although there was no significant difference.

Evaluation of IHC for PTEN. Fig. 1 shows the immunostaining patterns of PTEN. The cases shown are $3+/ 2+, 1+$ and negative. As shown in Fig. 2 and Table II, the immunostaining was evaluated as SI x PP using the H score. The H score was widely distributed ranging from 0 to 200 . The median values were 110,90 and 50 in groups A, B and C, respectively. Two patients in group $\mathrm{C}$ had a score of 0 . As shown in Table II, a decreased 
Table I. Background patient characteristics.

\begin{tabular}{|c|c|c|c|c|}
\hline Characteristics & Group A & Group B & Group C & P-value \\
\hline No. of cases & 15 & 7 & 11 & \\
\hline \multicolumn{5}{|l|}{ Age at registration (years) } \\
\hline Median & 60.5 & 60.0 & 47.0 & 0.03 \\
\hline Mean & 58.3 & 60.1 & 49.7 & \\
\hline \multicolumn{5}{|c|}{ Tumor size (primary tumor) $(\mathrm{cm})$} \\
\hline$\leq 5.0$ & $11(73.3)$ & $7(100)$ & $8(72.7)$ & 0.31 \\
\hline$>5.0$ & 2 & 0 & 3 & \\
\hline Unknown & 2 & 0 & 0 & \\
\hline \multicolumn{5}{|l|}{ Nodal status (primary tumor) } \\
\hline No & $4(26.7)$ & $1(14.3)$ & $2(18.2)$ & 0.79 \\
\hline N1 & 6 & 5 & 6 & \\
\hline $\mathrm{N} 2,3$ & 2 & 1 & 3 & \\
\hline Unknown & 3 & 0 & 0 & \\
\hline \multicolumn{5}{|c|}{ Distant metastasis (at primary surgery) } \\
\hline Without & 11 & 7 & 9 & 0.32 \\
\hline With & 4 & 0 & 2 & \\
\hline \multicolumn{5}{|c|}{ No. of involved nodes (primary surgery) } \\
\hline 0 & $4(26.7)$ & $1(14.3)$ & $2(18.2)$ & 0.35 \\
\hline $1-3$ & 5 & 1 & 4 & \\
\hline$\geq 4$ & 3 & 5 & 3 & \\
\hline Unknown & 3 & 0 & 2 & \\
\hline \multicolumn{5}{|c|}{ Stage classification (at primary surgery) } \\
\hline I & 2 & 0 & 0 & 0.52 \\
\hline II & 8 & 6 & 7 & \\
\hline III & 1 & 1 & 2 & \\
\hline IV & 3 & 0 & 2 & \\
\hline Unknown & 1 & 0 & 0 & \\
\hline \multicolumn{5}{|l|}{ Histological type } \\
\hline Invasive ductal carcinoma & 14 & 7 & 9 & 0.99 \\
\hline Others & 1 & 0 & 2 & \\
\hline \multicolumn{5}{|l|}{ Estrogen receptor } \\
\hline+ & $8(53.3)$ & $5(71.4)$ & $2(18.2)$ & 0.06 \\
\hline- & 7 & 2 & 9 & \\
\hline \multicolumn{5}{|l|}{ Progesterone receptor } \\
\hline+ & $5(33.3)$ & $1(14.3)$ & $2(18.2)$ & 0.53 \\
\hline- & 10 & 6 & 9 & \\
\hline \multicolumn{5}{|l|}{ HER2 } \\
\hline $1+, 2+$ & 1 & 1 & 2 & 0.66 \\
\hline $3+$ & 14 & 6 & 9 & \\
\hline \multicolumn{5}{|l|}{ HER2-FISH } \\
\hline Median & 5.2 & 8.6 & 4.6 & 0.34 \\
\hline Mean & 5.6 & 6.7 & 4.8 & \\
\hline \multicolumn{5}{|c|}{ Progression-free survival (days) } \\
\hline Median & 657 & 178 & 60 & $<0.0001$ \\
\hline Mean & 1151.8 & 174.4 & 103.3 & \\
\hline \multicolumn{5}{|l|}{ Disease-free interval (days) } \\
\hline Median & 409 & 491 & 337 & 0.21 \\
\hline Mean & 580.9 & 757.4 & 327.7 & \\
\hline
\end{tabular}


Table I. Continued.

\begin{tabular}{lcccc}
\hline Characteristics & Group A & Group B & Group C & P-value \\
\hline Operation performed for primary tumor & 14 & & 9 & 0.39 \\
Yes & 1 & 0 & 2 & 0.72 \\
No (advanced) & & & 3 & \\
Dominant site of recurrence & 4 & 2 & 2 & \\
Lymph node & 4 & 0 & 3 & \\
Bone & 3 & 1 & 2 & \\
Lung & 1 & 2 & 2 & \\
Liver & 2 & 0 & & \\
Chest wall & 1 & 0 & 3 \\
Locally advanced & 2 & & 7 \\
Other & & 1 & 1 \\
Timing of trastuzumab treatment & 2 & 6 & \\
Adjuvant setting & 12 & 1 & \\
1st or 2nd line after recurrence & 1 & 2 & \\
3rd line or later & & & & \\
\hline
\end{tabular}

HER2, human epidermal growth factor receptor 2; FISH, fluorescence in situ hybridization.

Table II. Immunohistochemistry for PTEN and the treatment efficacy of trastuzumab.

\begin{tabular}{lcccc}
\hline H score & Group A & Group B & Group C & P-value \\
\hline$\geq 100$ & $10(66.7)$ & $3(42.9)$ & $3(27.3)$ & 0.13 \\
$<100$ & 5 & 4 & 8 & \\
\hline
\end{tabular}

Cases with decreased expression (score <100): 17/33 (51.5\%).

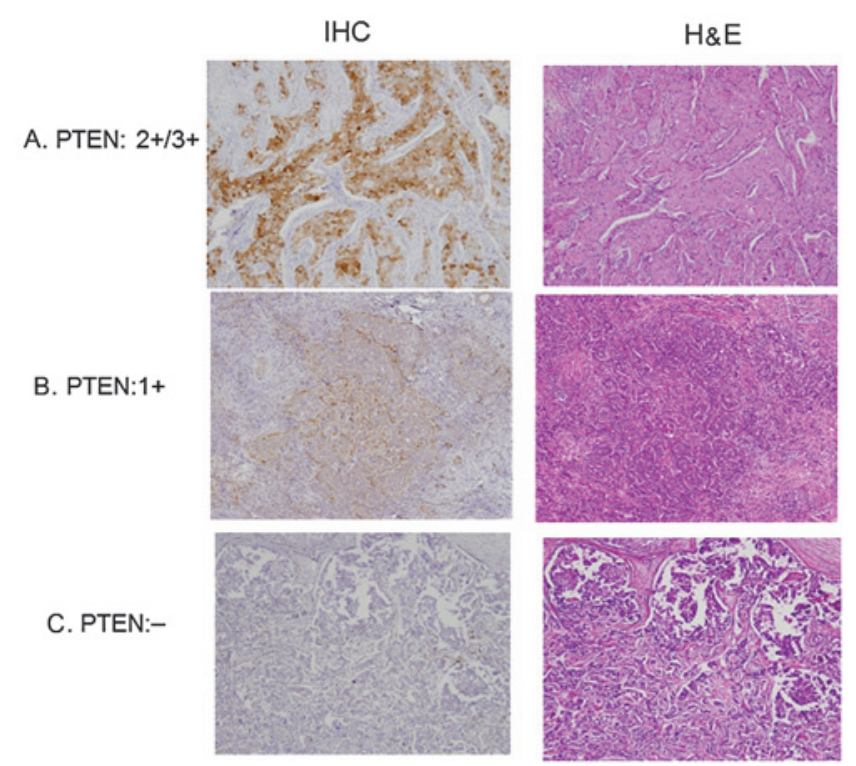

Figure 1. PTEN expression pattern in human breast cancers is shown. PTEN expression of the tumors was examined by immunohistochemistry (IHC) and evaluated using the $\mathrm{H}$ score. Representative tumor PTEN stainings and corresponding $\mathrm{H} \& \mathrm{E}$ stains (magnification, $\mathrm{x} 40$ ) are shown in $\mathrm{A}, \mathrm{B}$ and $\mathrm{C}$ as $2+/ 3+, 1+$ and -, respectively.
Table III. PIK3CA mutation and the treatment efficacy of trastuzumab.

\begin{tabular}{lcccc}
$\begin{array}{l}\text { PIK3CA } \\
\text { mutation }\end{array}$ & Group A & Group B & Group C & P-value \\
\hline Exon 9 & 2 & 0 & 1 & \\
Exon 20 & 3 & 0 & 2 & \\
Total & $5(33.3 \%)$ & 0 & $3(27.3 \%)$ & 0.23 \\
\hline
\end{tabular}

Cases with PIK3CA mutation: 8/33 (24.2\%).

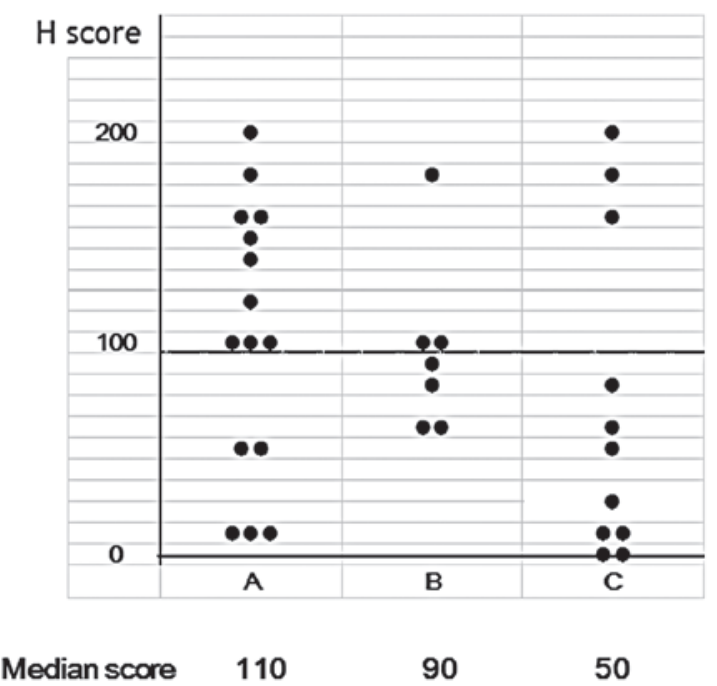

Figure 2. H score of immunostaining for PTEN, according to the efficacy of $\mathrm{T}$ treatment is shown. The $\mathrm{H}$ score is widely distributed from 0 to 200 . Median values are 110,90 and 50 in groups A, B and C, respectively. 
expression (score, <100) was observed in $17 / 33(51.5 \%)$ of the cases, when the patients were divided into 2 groups based on a $\mathrm{H}$ score of 100 . In group $\mathrm{A}$, the percentage of patients with a score of $\geq 100$ was $66.7 \%$, while in group $\mathrm{C}$ it was as low as $27.3 \%$. Although no statistically significant difference was detected, the number of patients with a decreased expression was high in group C. Consequently, PTEN immunostaining was assessed as described above, and was considered to have the potential to be used in the prospective study.

PIK3CA mutation. Table III shows the distribution of cases based on PIK3CA mutation. PIK3CA mutations were observed in $8 / 33$ (24.2\%), when mutations at exons 9 and 20 were combined. However, no clear correlation between the patient groups was observed in the distribution of patients based on the mutation.

\section{Discussion}

This retrospective study was conducted in preparation of a prospective study, aiming to investigate the correlation between the PFS of concomitant lapatinib/capecitabine therapy and the p95HER2 and PTEN protein expression levels, as well as the status of PIK3CA mutation in HER2-positive breast cancer patients.

To evaluate PTEN immunostaining, the $\mathrm{H}$ score was employed. According to available studies, evaluation of PTEN immunostaining by immunoreactive score (IRS) is common. Staining intensity (0-3) is evaluated on a 4-level scale. Percentage of positive cells (0-4) is evaluated on a 5-level scale of $0(<1 \%), 1(1-10 \%), 2(11-50 \%), 3(51-80 \%)$ and 4 (>80\% positive cells), based on the percentages of the positive cells. The entities use multiplied values (from 0 to 12 points). However, there are differences in the cut-off points, ranging from 3 to 8 points. The incidence of cases with decreased expressions also range from 19.1 to $51.8 \%$, showing a notable gap. In this study, the percentage of cases with a decreased expression was $51.5 \%$. However, the patients in this study were recruited according to the extent of response to $\mathrm{T}$, therefore additional investigation, intended to be carried out in the prospective study, is required.

With regard to P13KCA mutation, exon 9 or 20 mutation was found in $8 / 33(24.2 \%)$. Hennessy et al (20) reported the correlation between the frequency and breast cancer subtypes, while the mutation frequency was $9 / 19(47.4 \%)$ in metaplastic breast cancer patients, $232 / 880(34.5 \%$; $\mathrm{P}=0.32)$ in hormone receptor-positive patients, $17 / 75(22.7 \%$; $\mathrm{P}=0.04)$ in HER2positive patients, $20 / 240(8.3 \%$; $\mathrm{P}<0.0001)$ in basal-like patients and $0 / 14(\mathrm{P}=0.004)$ in claudin-low patients. According to the literature $(9-12,20)$, there was a clear correlation between PIK3CA mutation and the efficacy of T treatment. It was also reported (21) that $\mathrm{T}$ resulted in tumor regression subsequent to apoptosis induction, while not showing a significant change in cell proliferation, as measured by Ki-67. However, with lapatinib, both Ki-67 and p-MAPK levels showed a significant decrease during treatment. Thus, T seems to affect cell survival, while having a smaller effect on cell cycle kinetics, whereas lapatinib seems to affect cell cycle kinetics via RAS/MAPK, while having a smaller effect on cell survival (21). The present study, however, showed no clear correlation between this mutagenicity and the effects of T. Additional investigation in a prospective study is required regarding the correlation between this mutation and the anti-HER2 treatment effect.

With regard to p95HER2, an assay method for HER2 has been developed (22). This method uses two types of antibodies that bind to HER2. One of the antibodies binds by a fluorochrome linker, while the other is labeled by a chemical substance that generates a peroxidative enzyme when it is exposed to light and breaks the linker. When the two types of antibodies bind to HER2 on the cell surface, free fluorochrome is measured by capillary electrophoresis enabling the assay of the receptors. This process is termed VeraTag ${ }^{\mathrm{TM}}$ assay. This assay was also applied to p95HER2 (16). This method is now used in our ongoing prospective study. There were studies on IHC for p95HER2 that were presented at the annual meeting of the American Society of Clinical Oncology held in 2011. Although p95HER2 was suggested to be a marker of resistance to $\mathrm{T}$, in the neoadjuvant study by Loibl et al (23), there was a significantly higher rate of pathological complete response ( $\mathrm{pCR}$ ) by $\mathrm{T}$ treatment in cases that expressed p95HER2. In this study, the 611CTF monoclonal antibody (bioMérieux, Inc.) was used. Furthermore, even in the CHER-LOB trial (24), there was no clear correlation with the $\mathrm{T}$ and lapatinib treatments.

Looking at the p95HER2-positive rates in these studies, the values were $74 \%$ in the GeparQuattro study (23), 30.7\% in the CHER-LOB study (24) and $27.5 \%$ in the VeraTag ${ }^{\mathrm{TM}}$ assay (25). Although a difference was found between the early breast cancer and recurrent patients regarding the p95HER2 assessment, there was no constant trend, and there are a number of questions that remain to be solved. From these findings, the VeraTag $^{\text {TM }}$ method was set to be used to assess p95HER2 in our prospective study.

The immunostaining and assessment methods for PTEN expression were validated by the present study, and there was some correlation with the efficacy of the T treatment. However, for the PIK3CA mutation, there was no correlation with the $\mathrm{T}$ treatment results. This potential correlation is likely to be confirmed in the planned large-scale study, while the association of PIK3CA mutation and p95HER2 expression with poor response to $\mathrm{T}$ should also be examined.

\section{Acknowledgements}

The authors would like to thank the staff at the Department of Pathology in the Kumamoto City Hospital and the Division of Medical Oncology, Hematology and Infectious Diseases, of the Department of Medicine at the Fukuoka University, for their technical and secretarial assistance.

\section{References}

1. Harries M and Smith I: The development and clinical use of trastuzumab (Herceptin). Endocr Relat Cancer 9: 75-85, 2002.

2. Sáez R, Molina MA, Ramsey EE, et al: p95HER2 predicts worse outcome in patients with HER2-positive breast cancer. Clin Cancer Res 12: 424-431, 2006.

3. Molina MA, Sáez R, Ramsey EE, et al: NH2-terminal truncated HER2 protein but not full-length receptor is associated with nodal metastasis in human breast cancer. Clin Cancer Res 8: 347-353, 2002. 
4. Christianson TA, Doherty JK, Lin YJ, et al: NH2-terminally truncatedHER 2/neu protein: relationship with shedding of the extracellular domain and with prognostic factors in breast cancer. Cancer Res 58: 5123-5129, 1998.

5. Nahta R and Esteva FJ: HER2 therapy: molecular mechanisms of trastuzumab resistance. Breast Cancer Res 8: 215-222, 2006.

6. T Fujita, H Doihara, K Kawasaki, et al: PTEN activity could be a predictive marker of trastuzumab efficacy in the treatment of ErbB2-overexpressing breast cancer open. Br J Cancer 94: 247-252, 2006.

7. Nagata Y, Lan KH, Zhou X, et al: PTEN activation contributes to tumor inhibition by trastuzumab, and loss of PTEN predicts trastuzumab resistance in patients. Cancer Cell 6: 117-127, 2004.

8. Xia W, Husain I, Liu L, et al: Lapatinib antitumor activity is not dependent upon phosphatase and tensin homologue deleted on chromosome 10 in ErbB2-overexpressing breast cancers. Cancer Res 67: 1170-1175, 2007.

9. Pérez-Tenorio G, Alkhori L, Olsson B, et al: PIK3CA mutations and PTEN loss correlate with similar prognostic factors and are not mutually exclusive in breast cancer. Clin Cancer Res 13: 3577-3584, 2007.

10. Berns K, Horlings HM, Hennessy BT, et al: A functional genetic approach identifies the PI3K pathway as a major determinant of trastuzumab resistance in breast cancer. Cancer Cell 12: 395-402, 2007.

11. Toi M, Iwata H, Fujiwara Y, et al: Lapatinib monotherapy in patients with relapsed, advanced, or metastatic breast cancer: efficacy, safety, and biomarker results from Japanese patients phase II studies. Br J Cancer 101: 1676-1682, 2009.

12. Xu BH, Jiang ZF, Chua D, et al: Lapatinib plus capecitabine in treating HER2-positive advanced breast cancer: efficacy, safety, and biomarker results from Chinese patients. Chin J Cancer 30: 327-335, 2011.

13. Maruyama N, Miyoshi Y, Taguchi T, Tamaki Y, Monden M and Noguchi S: Clinicopathologic analysis of breast cancers with PIK3CA mutations in Japanese women. Clin Cancer Res 13: 408-414, 2007.

14. Saal LH, Holm K, Maurer M, et al: PIK3CA mutations correlate with hormone receptors, node metastasis, and ERBB2, and are mutually exclusive with PTEN loss in human breast carcinoma. Cancer Res 65: 2554-2559, 2005.

15. Scaltriti M, Rojo F, Ocaña A, et al: Expression of p95HER2, a truncated form of the HER2 receptor, and response to anti-HER2 therapies in breast cancer. J Natl Cancer Inst 99: 628-638, 2007.

16. Sperinde J, Jin X, Banerjee J, et al: Quantitation of p95HER2 in paraffin sections by using a p95HER2-specific antibody and correlation with outcome in a cohort of trastuzumab-treated breast cancer patients. Clin Cancer Res 16: 4226, 2010.
17. Hayashi M, Okumura Y, Osako T, Toyozumi Y, Arima N, Iwase $\mathrm{H}$ and Nishimura R: Time to first tumor progression as a predictor of efficacy of continued treatment with trastuzumab beyond progression in human epidermal growth factor receptor2positive metastatic breast cancer. Int J Clin Oncol 16: 694-700, 2011.

18. Metro G, Giannarelli D, Gemma D, et al: Time to first tumor progression as outcome predictor of a second trasuzumab-based therapy beyond progression in HER-2 metastatic breast cancer. Breast J 16: 66-72, 2010.

19. Shousha S: Oestrogen receptor status of breast carcinoma: Allred/H score conversion table. Histopathology 53: 346-347, 2008.

20. Hennessy BT, Gonzalez-Angulo AM, Stemke-Hale K, et al: Characterization of a naturally occurring breast cancer subset enriched in epithelial-to-mesenchymal transition and stem cell characteristics. Cancer Res 69: 4116-4124, 2009.

21. Dave B, Migliaccio I, Gutierrez MC, et al: Loss of phosphatase and tensin homolog or phosphoinositol-3 kinase activation and response to trastuzumab or lapatinib in human epidermal growth factor receptor 2-overexpressing locally advanced breast cancers. J Clin Oncol 29: 166-173, 2010.

22. Yining $\mathrm{S}$, Weidong $\mathrm{H}$, Yuping $\mathrm{T}$, et al: A novel proximity assay for the detection of proteins and protein complexes: quantitation of HER1 and HER2 total protein expression and homodimerization in formalin-fixed, paraffin-embedded cell lines and breast cancer tissue. Diagnostic Molecular Pathology 18: 11-21, 2009.

23. Loibl S, Bruey J, Von Minckwitz G, et al : Validation of p95HER 2 as a predictive marker for trastuzumab-based therapy in primary HER2-positive breast cancer: a translational investigation from the neoadjuvant GeparQuattro study. J Clin Oncol 29: 530, 2011.

24. Guarneri V, Frassoldati A, Bottini A, et al: Final results of a phase II randomized trial of neoadjuvant anthracycline-taxane chemotherapy plus lapatinib, trastuzumab, or both in HER2-positive breast cancer (CHER-LOB trial). J Clin Oncol 29: 507, 2011.

25. Biernat W, Duchnowska R, Szostakiewicz B, et al: Quantitative measurements of p95HER2 (p95HER2) and total HER2 (H2T) protein expression in patients with trastuzumab-treated, metastatic breast cancer (MBC): Independent confirmation of clinical cutoffs. J Clin Oncol 29: 586, 2011. 